\title{
SEMANTICALLY DOCUMENTING VIRTUAL RECONSTRUCTION: BUILDING A PATH TO KNOWLEDGE PROVENANCE
}

\author{
G. Bruseker ${ }^{\mathrm{a}}$, A. Guillem ${ }^{\mathrm{b}}$, N. Carboni ${ }^{\mathrm{c}}$ \\ ${ }^{a}$ FORTH - Centre for Cultural Informatics, Heraklion, Greece - bruseker@ics.forth.gr \\ ${ }^{\mathrm{b}}$ Faculty of Civil and Geodetic Engineering, University of Ljubljana, Ljubljana, Slovenia - anais.guillem@fgg.uni-lj.si \\ ${ }^{\mathrm{c}}$ UMR 3495 MAP CNRS/MCC, Marseille, France - nicola.carboni@map.cnrs.fr
}

KEY WORDS: Virtual Reconstruction, Reconstruction Process, Reasoning Process, Modeling, Provenance, CIDOC-CRM

\begin{abstract}
:
The outcomes of virtual reconstructions of archaeological monuments are not just images for aesthetic consumption but rather present a scholarly argument and decision making process. They are based on complex chains of reasoning grounded in primary and secondary evidence that enable a historically probable whole to be reconstructed from the partial remains left in the archaeological record. This paper will explore the possibilities for documenting and storing in an information system the phases of the reasoning, decision and procedures that a modeler, with the support of an archaeologist, uses during the virtual reconstruction process and how they can be linked to the reconstruction output. The goal is to present a documentation model such that the foundations of evidence for the reconstructed elements, and the reasoning around them, are made not only explicit and interrogable but also can be updated, extended and reused by other researchers in future work. Using as a case-study the reconstruction of a kitchen in a Roman domus in Grand, we will examine the necessary documentation requirements, and the capacity to express it using semantic technologies. For our study we adopt the CIDOC-CRM ontological model, and its extensions CRMinf, CRMBa and CRMgeo as a starting point for modelling the arguments and relations.
\end{abstract}

\section{INTRODUCTION}

In a media focused environment, where the knowledge and tools for the production of realistic, even hyper-realistic, images of reconstructed archaeological sites and monuments in two, three and four dimensions attracts a growing and diverse audience, the questions regarding the maintenance of a scholarly foundation of the virtual reconstruction process are a pressing concern. In ideal circumstances, it would involve the interdisciplinary cooperation of at least an archaeologist and a modeler working together to produce a probable reconstructed whole of some point in the past, founding their work on retrievable evidence and argumentation (Pletinckx, 2008). In practice, regardless whether such cooperation is undertaken and such reasoning applied, the process that leads to the virtual reconstruction outcome often stands only as an outcome and conclusion in the visual product that does not answer the question of the why or how of its production, but only of the what. It leaves the audience without a real explanation of what are they seeing and therefore fails in important ways in its explanatory purpose. As De Luca and Buglio (2014) clearly state, "if the (constantly growing) mass of data has effectively enabled to approach the reconstruction of complex geometries, this overgrowth (of data) does not seem to increase the level of intelligibility of the representations produced." It is an outcome of this situation not only that this undocumented reasoning process is lost to the scientific community for interrogation and future reuse but, further, an important means of distinguishing the realistic against the hyper-real, the founded versus the propagandistic image, is undermined (Baudrilliard, 1995; Eco, 1990; Hermon \& Kalisperis, 2011). Documents like the London Charter (Denard, 2009) and the Seville Principles (LopezMenchero \& Grande, 2011) offer guidelines and directions for what proper documentation might look like; still, it remains for the professionals working in this area to devise the actual means to sustainably achieve these admirable principles. Towards this aim, advances in semantic representation of argumentation offer the possibility of a fruitful collaboration between professionals in knowledge management and virtual reconstruction to devise accurate and efficient means to document these processes.

In this paper, we make a step in this direction, taking a case study of a reconstruction of a kitchen in a Roman domus in Grand, and analyzing the essential components of the process starting with the commissioning of the project and concluding with the transfer of the digital outcome back to the commissioner. Adopting the cultural heritage ontology CIDOCCRM (Le Bœuf, Doerr, Ore, \& Stead, 2015) and, in particular, its extension CRMinf (Paveprime Ltd. 2014), on argumentation documentation, we set out to document the minimum steps and reasoning processes involved in the generation of a virtual reconstruction. CIDOC-CRM is the ISO 21127:2014 formal model for describing cultural heritage developed by CIDOC, the International Committee for Documentation of the International Council of Museums (ICOM). CRM stands for Conceptual Reference Model. CRMinf stands for CRM Inference and is the acronym for the argumentation model. The outcome is a highlevel, generic process model that documents the reasoning behind such a process and allows it to be linked both to its digital outcome and to elements of an information system storing data regarding the site or monument under reconstruction. 


\section{PRINCIPLES AND STANDARDS OF KNOWLEDGE PROVENANCE IN VIRTUAL RECONSTRUCTION}

In this section of the paper, we frame our work in relation to the principles and standards we adopt in the task of creating knowledge provenance in virtual reconstruction.

\subsection{Principles: London Charter and Seville Principles}

Our arguments in this paper are guided by the principles set out in the London Charter and the Seville Principles. Here we aim not to provide an exhaustive review of the state-of-the-art on the question of transparency in virtual reconstruction but rather to pick out some of the most relevant elements for founding our thesis and to give an overview of our framework.

The London Charter is an international charter dedicated to computer-based visualisation of cultural heritage. Directly in the preamble, it lays out the most basic goal: to "ensure that digital heritage visualisation is, and is seen to be, at least as intellectually and technically rigorous as longer established cultural heritage research and communication methods'. The Charter uses as a foundational premise that 'the outcomes of research (...) should accurately convey to users the status of the knowledge that they represent, such as distinctions between evidence and hypothesis, and between different levels of probability" (Denard, 2009). Later, in the section concerning documentation, the Charter highlights the necessity that, "computer-based visualisation outcomes should be disseminated in such a way that the nature and importance of significant, hypothetical dependency relationships between elements can be clearly identified by users and the reasoning underlying such hypotheses understood." (Denard, 2009). These three short quotations show the critical challenge of documenting the arguments and evidence deployed in the process of cultural heritage study.

While the London Charter points out the generic need of transparency in the relevant fields of cultural heritage, the Seville Principles take up these reflections and examine their implementation in the field of virtual archaeology. They propose a definition of virtual reconstruction as "using a virtual model to visually recover a building or object made by humans at a given moment in the past from available physical evidence of these buildings or objects, scientifically-reasonable comparative inferences and in general all studies carried out by archaeologists and other experts in relation to archaeological and historical science" (Lopez-Menchero \& Grande, 2011). Such a definition is a clear encouragement to more formally document the process of reconstruction activities in order to make explicit their meaning.

\subsection{Standards: CIDOC-CRM}

The adoption of these principles poses the immediate question of what internationally recognized standards we can use to express them. Any effort to transparently document the reconstruction process must, by definition, be expressed in a manner that allows the greatest uptake, accessibility and reusability by the broadest audience possible. Additional to these central requirements, further necessary considerations for the adoption of a standard for knowledge representation are the ability to express the reasoning over parts and wholes that is at the core of the virtual reconstruction analysis, and its capability to represent and store this argumentation. For these reasons, we adopted CIDOC-CRM as the knowledge representation tool by which to undertake our process modeling.

The CRM responds well to the need to reach the greatest possible audience in a sustainable way. The primary audience to be addressed are cultural heritage professionals considered in the broadest sense: from archaeologists to exhibition designers to building restoration technicians. CRM is the outcome of almost two decades of continuous research in semantic representation in the field of cultural heritage (Oldman, Doerr, de Jong, Norton, \& Wikman, 2014). It has wide adoption and recognition as both a conceptual and information sharing tool and increasingly as the basis for the production of information systems implementations. A mark of its growing reach is the expansion of CRM compatible models dealing with the more detailed expression of sub-fields of the cultural heritage domain from which we were able to adopt elements, in particular: CRMgeo (Doerr \& Hiebel, 2013) for spatial information, CRMdig (Doerr \& Theodoridou, 2014) for digitization information and others to be explored below. Further, the implementation neutral stance of CRM makes it particularly suitable to our task. The present model is built as a proposed guide for the elaboration of actual systems, but, in order to enable adoption by the greatest audience possible, does not define or determine by which means such systems should be implemented

\subsubsection{Reasoning over parts and whole in virtual reconstruction: CRM and CRMBa}

From the point of view of expressivity, the necessary condition for any representational system that would capture virtual reconstructions is the ability to represent reasoning over parts and wholes. Research into the ability to express this type of reasoning process has been undertaken since early in the development of the CRM. In their (1999) work, Bekiari and Doerr proposed a methodology of conceptual modeling of data to document incomplete knowledge about parts and wholes. In that and subsequent work they examine the formalization of the process of reconstructing parts from wholes with archaeological objects as a sample case. Applying mereological principles, they look at reconstructing wholes based on reasoning from different types of parts and from categorical knowledge of kinds of parts to kinds of wholes. Building on this work, Paola Ronzino recently proposed an extension to CRM called CRMBa (Ronzino, 2015) for supporting documentation in the field of building archaeology (Schuller, 2002). This extension focuses on the analysis of the parts of a building and their mereological and mereotopological relations, defining a new set of entities and relations useful in our analysis. Ronzino introduces a complex built element (Built Work), composed by morphological sections that can be broken down into filled or empty spaces and can be related to particular functions. We are able to take preliminary advantage of this work in our model by 
linking propositions from the virtual reconstruction process the overall built work and elements that make it up.

\subsubsection{Knowledge Provenance: context and CRMinf}

Since the question of knowledge provenance lies at the heart of data transparency and, therefore, the goals of our model, it would be useful to take a step back now and view the problem in context, and the particular response that CRMinf provides. The accurate tracing of the origin of digital sources of information has been a vibrant area of investigation in different fields for several decades. The subject's importance has grown in relation to the quantity of stored information, as well as its value from a scientific and legal point of view. Although analogous issues had already been faced even in the XIX century (Sweeney, 2008), the importance of the concept of knowledge provenance has grown exponentially in recent years, thanks to the exploding body of digital information (Hilbert \& López, 2011; Marins, Casanova, Furtado, \& Breitman, 2007).

The problem has been faced in different ways in the several fields in which it has been addressed. For example, while in computer science the focus has been mostly on where data comes from and why it appears in query results (Khanna, Tan, \& Buneman, 2001), in the GIS field, the emphasis has been on transformations applied during data creation (Simmhan, Plale, $\&$ Gannon, 2005). These different approaches, however, have recently started to converge on a broad definition of the problem, (Missier, Belhajjame, \& Cheney, 2013) that defines and divides the issue in terms of: agent-centered provenance, object-centered provenance, and process-centered provenance. For the purposes of this paper, our main interest is the last of these. Insofar as we touch on object-centred provenance issues, we adopt the methodology and point of view proposed in CRMdig (Doerr \& Theodoridou, 2011; Tzompanaki, Doerr, \& Theodoridou, 2013).

Process-centered provenance tracking takes into consideration the mapping of a series of statements that explain the transition from the premises to the conclusion of an argument. It is used to show the differences and choices in data creation and elaboration. This kind of information helps to define the value of the data and can be used to enable the reuse of reasoning chains, both from a legal and from a scientific perspective, in addition to being helpful in identifying the context of information as a crucial aspect in the preservation of the identity of a dataset (Doerr \& Tzitzikas, 2012).

The formalisation of process-centered provenance has been underway for the last thirty years. One of the first contemporary examples is IBIS (Issue-Based Information System) (Kunz \& Rittel, 1970), an argumentation model developed to support planning in the political process. It focuses on issues, defined by questions, that are resolved through ideas that carry some specific arguments. A major drawback of this model for our purposes is that it doesn't allow for concatenating inferences because it only focuses on problem solving in planning and support.
Another research field that has extensive experience with such tools is artificial intelligence (Reed, Walton, \& Macagno, 2007) where models like the argumentation framework proposed by Dung (1995) were developed in order to help machines not simply understand natural language interactions but also help AI in interpreting arguments. Argumentation modeling for legal reasoning can even be traced back to the early 1930s when John Henry Wigmore develop such models for structuring evidential reasoning to help decision-making in law-related cases. (Bex, Prakken, Reed, \& Walton, 2003). For a longer review of such models, see Doerr, Kritsotaki, \& Boutsika (2011).

Gardin, one of the main supporters of such models in the cultural heritage field, has advocated that such formalizations be applied to archaeological argumentation so as to display their, "constituents in a primitive logical form that helps to apprehend the overall organisation of the interpretation process and to consult readily some of its parts without having to go through lengthy presentations in standard archaeological discourse" (Gardin, 2002). One response to this call is made in the integrated argumentation model described in Doerr, Kritsotaki, \& Boutsika (2011). A simplified version of this model has been implemented in CRM and called CRMinf. It works on the principle of documenting chains of reasoning that support beliefs in some state of knowledge. It stores the propositions used and the logics applied or sources referred to in order to support these claims. Further, it does so in a manner that is integrated to the CIDOC-CRM model. It was thus deemed an ideal model to test for the scenario of virtual reconstruction as an activity that works from partial evidence and leads to conclusions on hypothetical whole reconstructions.

Modeling virtual reconstruction as a reasoning process based on a series of propositions, as we propose to do in our model, promises a distinct advantage in the retrievability of the provenance of knowledge. First, the propositions identified are traceable back to their source, second, the propositions can be examined based on their relation to the catalogued elements of an information system representing the physical evidence and remains of an archaeological built space. The propositions are 'about' something. The elements described can be remeasured and therefore the propositions can be reassessed. New evidence can be introduced at any time. The representation of the reasoning chain allows for tracing which conclusions downstream would then need to be reassessed, and which premises upstream, where conflicts originate, would necessarily trigger an act of reanalysis. Additionally, this serves as an intellectual check on any eventual digital representations that may be produced out of the reconstruction process. They, too, are linked back to the objects through the propositions in order to verify their representational accuracy. Taken together, these links offer the potential of a highly versatile path of knowledge, allowing one to move from the catalogued remains through to their propositional representation in documentation, all the way to their propositional display in any number of virtual reconstructions. This path can be traversed starting from any position in the graph. 


\section{PRESENTATION OF THE MODEL}

\subsection{Case study of the kitchen's domus at Fontainotte (Grand, France)}

In order to build our model of virtual reconstruction, we take as a case study the domus kitchen at Fontainotte (Grand, France). This study is a previous work, an illustration for the new exhibition of the Musée de la Mosaïque that was commissioned in relation to a renovation programme to facilitate visitor access. The permanent exhibition was renewed and it was decided to present some of the results of the excavation of Fontainotte's domus. To facilitate the visitor's understanding of the remains, objects and documents, a reconstruction illustration was ordered in 2013 by the curator of the site, Th. Dechezleprêtre, and the new exhibition designer, architect M. Kintz. The excavation was conducted by INRAP (Institut National de Recherche en Archéologie Préventive) under the supervision of M. Gazenbeek in 2011. The reconstruction illustration aimed at dissemination of recent archaeological results/research to the general public, with a focus on adults and youth, as a medium of representation between the objects and the guides' explanations.

The reconstruction illustration was ordered before the publication of the official report of the whole domus excavation. Nevertheless, Gazenbeek's article (2013) offers a synthesis of the scientific results already obtained with regards to the domus' kitchen. At each step of the process, M. Gazenbeek (archaeologist supervisor of the excavation) and Th. Dechezleprêtre (director of the site and curator of the museum) were consulted for modifications, advice and questions. The result is a black and white line drawing representing a view on the kitchen from the doorstep of the kitchen's space. The perspective adopted was chosen to be as close as possible to that which an average person would have on entering the space. We chose this illustration for the case study as a relatively ideal prototype: a one-room space whose archaeological elements (fireplace, cooking objects, food left-over, archaeological structures, etc) are well documented and well researched. This allows us to represent at least an ideal workflow of the basic steps in reconstruction though, with elaboration, this model could certainly be extended to more complex spaces and buildings.

\subsection{Model}

In the model analytically presented below, we consider each phase of the virtual reconstruction process as a discrete set of activities whose output constrains the next phase of activities. The process is deemed to begin with the commissioning from some party and is considered complete with its production and delivery to the commissioning agent. It is a process that shares common functions with other scholarly pursuits - research, analysis and reasoning phases - in order to arrive at its end result. We consider the outcome of this activity to be a reasoned representation of a three dimensional living volume presented for some specific audience that is based on the synthesis of factual and hypothetical evidence by a particular modeler at some time. It is because of and insofar as the virtual reconstruction process arrives at its conclusions - propositions supporting the restoration of some partial element as a complete whole - through a chain of reasoning founded on premises believed to be true, and through executing reasonable logical inferences that we can treat its outcome as an argument with strengths and weaknesses open to critical examination. We argue that only by capturing the sources used, their relations, as well as the logic applied to the process, can the virtual reconstruction properly become a scientific object within the scholarly discourse.

Our model presents a semantic representation of the above process in eight phases. We argue that this model is sufficient to document the most relevant factors that inform and support the creation of the final reconstruction. The phases we model are: commissioning, documentation research, proposition identification, function hypothesis assumption, global geometric volume reasoning, in-situ element reconstruction, ex-situ element reconstruction, and visual representation production.

The further elaborated the reasoning becomes through the process, the further constrained the reasoning choices are that are left available to the modeler. This constraining role serves a positive function in using previously secured evidence to act as a limit to hypothetical assumptions, thus strengthening the probability of any given hypothetical argument to the whole. The process is not, however, unidirectional. For each of the reasoning phases - global geometric volume reasoning, in-situ element reconstruction, ex-situ element reconstruction - the reasoning process is looped, both relative to the domain of objects of its concern and, if necessary, returning to previous phases and reconsidering premises and conclusions that do not fit following further reasoning. In what follows we provide a description of each phase with regards to its function, the inputs that support it and the outputs it generates.

Commissioning Phase: The reasoning process is initiated by the commissioning by some party of a modeler to build a virtual reconstruction for some audience. This audience, for whom the final result is intended, affects the choices of the modeler in terms of the focus and aim of the reconstruction. The same space will not be reconstructed in the same way for an audience of school children as it will for the specialist, though both representations are entirely reasonable outcomes of a principled modeling process. Therefore, the audience constraint is introduced as a first framing limitation passed into the process. The participation of a domain specialist, such as an archaeologist, is envisioned as constitutive to the entire process, providing much of the data and knowledge from which the model will proceed. Additional external restrictions could also include funding and time constraints. Here, however, we do not take the latter into consideration since it is not strictly part of the abstract reasoning process.

Documentation Research: When the commission is accepted, the modeler initiates a phase of documentation research. This phase entails the gathering and ordering of a series of relevant documents that will support the reasoning process used for conceptualizing a reconstructed whole, according to the usual 
ISPRS Annals of the Photogrammetry, Remote Sensing and Spatial Information Sciences, Volume II-5/W3, 2015

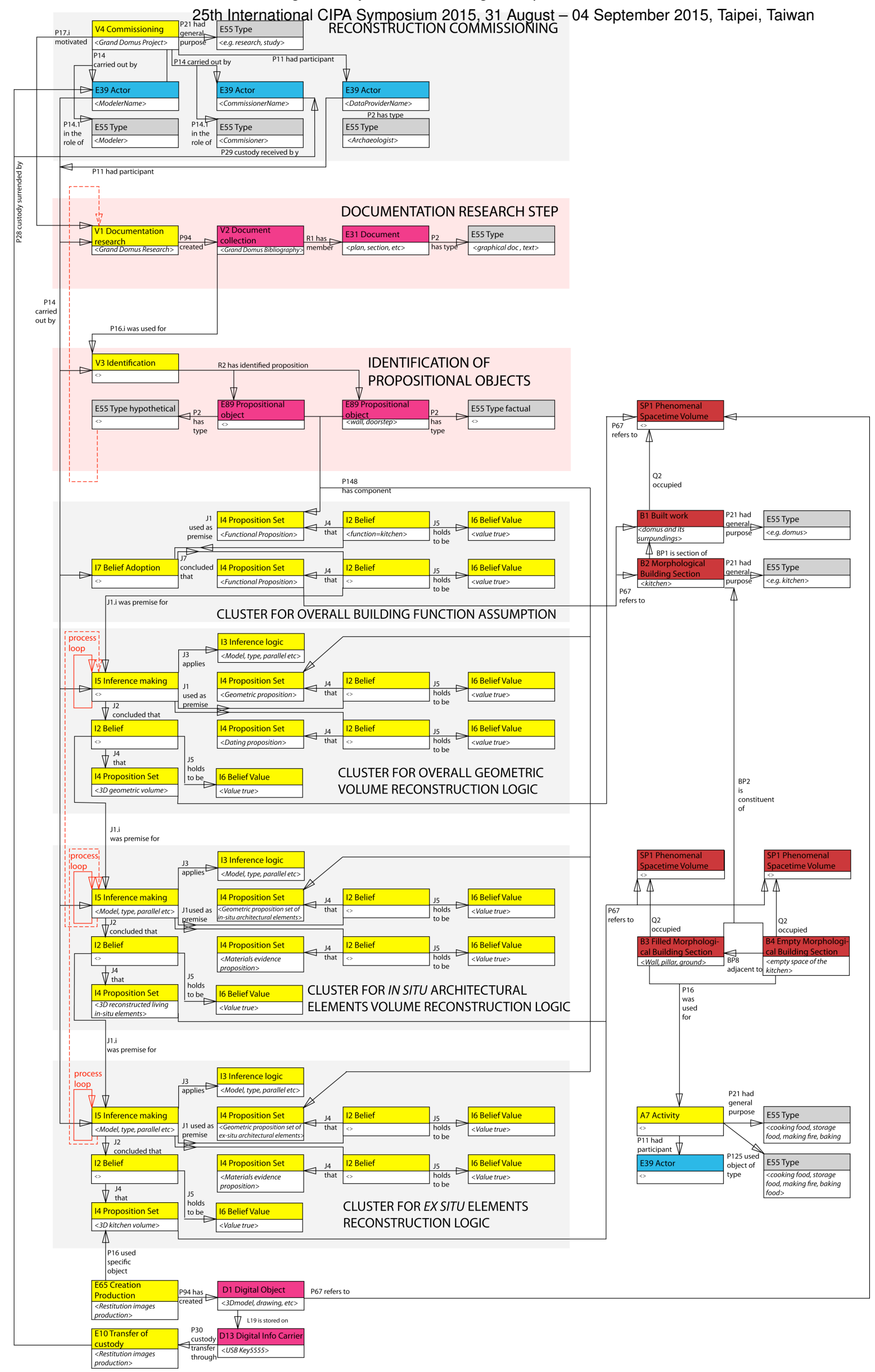

Figure 1. Graphical presentation of the virtual reconstruction process and argumentation model

This contribution has been peer-reviewed. The double-blind peer-review was conducted on the basis of the full paper. 
norms of academic activity. The documents gathered in this phase may include plans, maps, reports and articles related to the built space in question. Such a collection may further include general literature, thesauri and other reference materials relevant to the wider cultural and architectural field in which the space in question can be considered. The output of this activity establishes a documentation collection with an identifiable membership. These resources act as the next constraining factor to the process, as well as a source for the reasoning undertaken in following phases as they serve as the identifiable and reconstitutable knowledge pool from which the modeler drew her conclusions.

Proposition Identification: Having gathered a documentation resource pool, the modeler goes through a process of identifying the relevant information for her project. This activity is, in fact, a process of proposition identification that isolates those propositions that relate to the geometric space, individual elements and the whole structure under consideration. These propositions might be in the form of sentences as much as in the form of images, a passage stating the dimensions of the element or a plan indicating the same. The propositions identified can be further broken down in two different classes: factual and hypothetical. The former result from the process of a measurement of some existent remain and represent a best approximation of an empirical reality encountered through an observation at some point in time. The latter are the result of the argumentation of the authors of the documentation sources and deliver propositions based on observation plus some reasoning. The proposition sets gathered during this phase of the reconstruction, and identified with regards to their factual and hypothetical status, provide the constraints for the next phase of the process, and act as the necessary input for the reasoning cycles to come.

Building Function Assumption: With the above propositions in place, the intellectual process of planning the reconstruction commences. This phase involves commitment to recreating the built space as a certain kind of functional space. In other words, it involves the modeler in the commitment to a belief that the space had a certain dominant operational status during the time period which the modeler is tasked to represent. It is essential to document this step since it operates as a global reasoning constraint upon the rest of the reconstruction process, directly affecting the overall field of categories and objects by which the modeler can thereafter reason. The building function assumption informs the range of reconstruction options available to the modeler in terms of typical structures and equipment relative to this activity space. Assuming the function of kitchen for a space gives an entirely different range of articulation of representation choices to the modeler than would the assumption of a salon. The social-functional space provides the reason for which actual structures were put in place and thus is determinative of all following choices.

We model the determination of the building function assumption as a case of belief adoption in the CRMInf argumentation model. While it is certainly the case that inferential reasoning can, and must, be made to arrive at a conclusion on the function of a building known only from archaeological remains, the most likely scenario with regards to the modeler is that the space has already been determined by the commissioning agent, who has asked them to reconstruct an ' $\mathrm{X}$ ' type structure. For that reason we model this phase as a logical function of belief adoption.

Geometric Volume Reconstruction Logic: The next unit of reasoning that has to be undertaken is the determination of the overall geometric volume in which the reconstruction will take place. This part of the argumentation process adopts the building function assumption, and brings into consideration the geometric and dating propositions identified in the sources. These three sets of propositions can be reasoned upon to come to a conclusion on the overall geometric volume of the reconstruction space. The modeler can apply logics of geometry, type, and parallel to work out a most probable volume. We model this proposition as being about the overall space-time volume of the built space in question, since it is founded on evidence with regards to the total geometric and temporal volume of the built space during a certain phase of its existence. The statements are about the geometric volume but as a functional lived space at some time. In the current phase, and in the following ones, we envision the participation of an archaeologist, or another data provider designated by the commissioner of the reconstruction, as a potential participant in the reasoning process. The act of checking back with the specialist allows the external examination of the reasoning process and the introduction of other arguments.
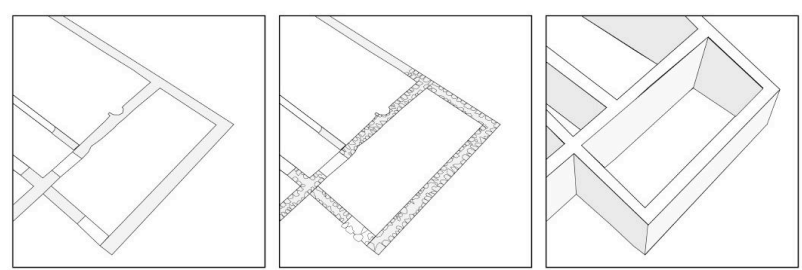

Figure 2. Reconstruction of the the geometric volume

In-situ Elements Reconstruction Logic: Having defined the overall spatio-temporal volume of the structure, the actual elements of the structure can now begin to be built up. This process involves a reasoning loop which takes as its inputs the geometric propositions regarding in-situ elements and the materials that compose them together with the preceding propositions as constraints. This follows the principles of working from the most certain elements first and applies the same logics as above, in order to reconstitute the whole elements of the building from the anchor points of the in-situ partial remains. Whole parts are reconstituted according to their constituency in the overall building or morphological building section, fitting filled and empty morphological building sections to the whole. Again, we represent the concluding propositions as referring to the overall space-time volume of the individual elements, with the idea in mind that conclusions will be about the overall life of the morphological building section for a 
phenomenal period of time. The reconstitution of whole building sections set limits to and establishes connection points upon which to rebuild ex-situ and entirely missing elements in the final reconstruction reasoning phase
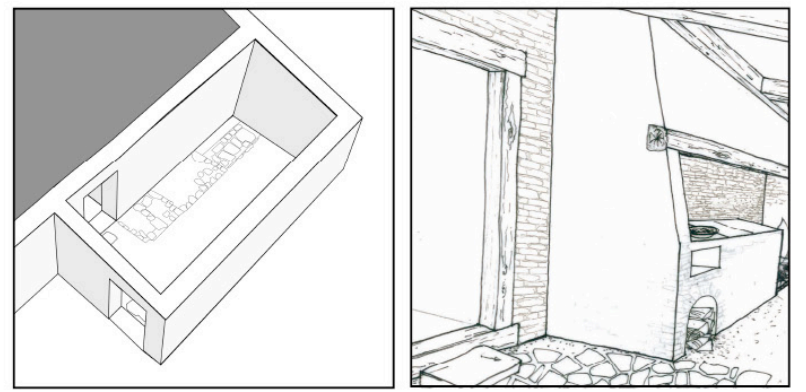

Figure 3. In-situ element reconstruction

An increase in the amount of evidence, and evidence types, along with complimentary inferential arguments strengthens the probability of the proposed reconstruction of the element. This phase of reconstruction may also trigger reconsiderations of the previous phase. Conclusions on some element of the built space may challenge the conclusion on the overall built space and trigger the need to revisit and readjust the previous conclusions.

Ex-situ Elements Reconstruction Logic: With the geometric volume defined, and filled or empty building sections of the overall building or general building section reconstituted on the basis of the in-situ evidence, there remains the restitution of exsitu and missing elements. At this point in the overall reasoning process, the space has been limited many times over, thus making the inference operations executed on the final, least certain elements as strong as possible by establishing them in relation to better established propositions and outcomes. For the remaining missing whole elements of the building, the modeler executes a round of analysis, applying the known logics and recording their results as conclusions on the space-time volume of building sections now reconstituted based largely on hypothesis and constraining evidential factors. Again, this process may result in the need for revision of previous phases if no reasonable conclusion fitting to previous conclusions can be found.
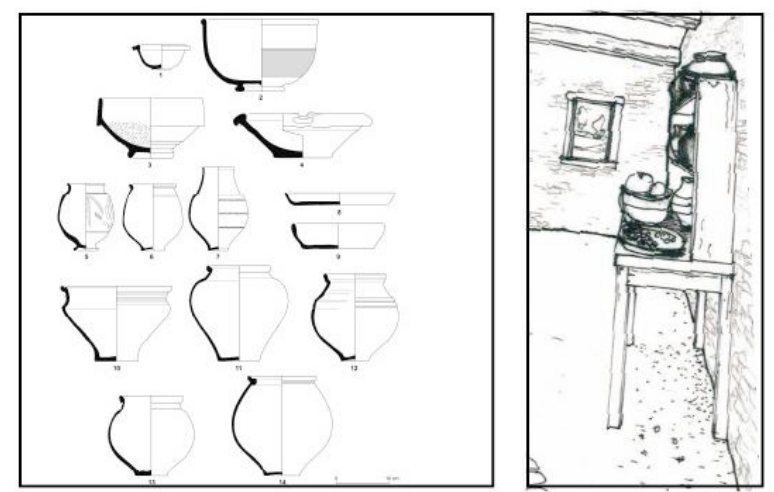

Figure 4. Ex-situ element reconstruction
Reconstruction Production: the outcome of the above procedures is sufficient to form a complete plan for the execution of a virtual reconstruction, an act of intellectual creation that finds its expression, normally, in a digital product (e.g.: raster or vector image, 3D model, 2D plan file etc.). The execution of a reconstruction process under the aegis of such a procedure would result in an outcome that would stand as both a rigorous intellectual argument, with regards to its spacetime volume, and as a path to knowledge connecting evidence, documentation and argumentation in a queryable information graph. We model the completion of the process as being constituted by the transfer of custody of the commissioned digital object from the modeler to the original commissioner.

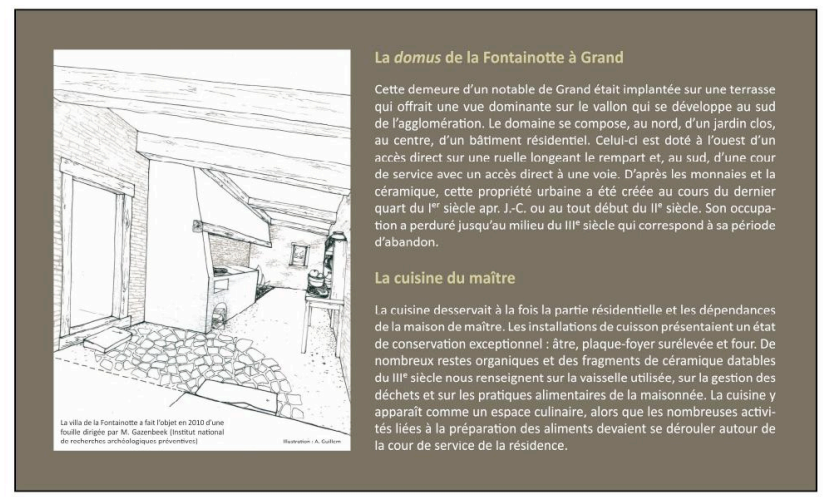

Figure 5. Final outcome, integration of the image in an exhibition's sign

\section{CONCLUSION}

In this paper, we set out to meet the knowledge provenance challenge set out by the London Charter and Seville Principles to link virtual reconstruction outcomes to their evidentiary and reasoning bases. Our proposed solution was a generic documentation model, which captures the essential steps of the process and their relations. Adopting the standard of CIDOCCRM and, in particular, its CRMinf and CRMBa extensions, enabled the expression of a generic level model that a) captured the decision and reasoning steps of the process, $b$ ) allowed these steps to be linked back to information systems about particular sites and monuments and c) connected the reasoning steps to the eventual digital model produced. We believe this model to be a substantial first step for further research in this area. While an implementation of this model would allow for a reasonable accuracy of documentation, our model does not presently analytically present information on important aspects of reconstruction including: probability strength of derived propositions, modeling of alternate reconstruction possibilities or an analytic decomposition of the inference logics particular to the field of virtual reconstruction. Further, the strength of the model could be tested by a broad selection of case studies to determine its overall adequacy. Nevertheless, we believe that the model presented above provides a useful starting point for consideration of implementable systems for the recording of the virtual reconstruction process, supporting these important intellectual works as they should be understood, as standing arguments, not mere pictures. 


\section{ACKNOWLEDGEMENTS}

This project has received funding from the European Union's Seventh Framework Programme for research, technological development and demonstration under grant agreement no 608013 .

This article would not have been possible without the support and the data of the archaeological site of Grand (Vosges, France) and its researchers' team under the supervision of $\mathrm{T}$. Dechezleprêtre, curator of MDAAC Museum in Epinal.

\section{REFERENCES}

Baudrillard, J. (1995). Simulacra and Simulation (17 ed.). Ann Arbor: University of Michigan Press.

Bex, F., Prakken, H., Reed, C., \& Walton, D. (2003). Towards a Formal Account of Reasoning about Evidence: Argumentation Schemes and Generalisations. Artificial Intelligence and Law, $11(2), 125-165$.

De Luca, L., \& Buglio, Lo, D. (2014). Geometry vs Semantics: open issues on 3D reconstruction of architectural elements. In M. Ioannides \& E. Quak, 3D Research Challenges in Cultural Heritage.

Denard, H. (2009). The London Charter for the Computer-based Visualisation of Cultural Heritage. King's College London.

Doerr, M., \& Hiebel, G. (2013). CRMgeo: Linking the CIDOC CRM to GeoSPARQL through a Spatiotemporal Refinement. ICS-FORTH.

Doerr, M., \& Theodoridou, M. (2011). CRMdig: A Generic Digital Provenance Model for Scientific Observation. Presented at the International Workshop on Theory and Practice of Provenace 2011.

Doerr, M., \& Tzitzikas, Y. (2012). Information Carriers and Identification of Information Objects: An Ontological Approach Doerr, M., Kritsotaki, A., \& Boutsika, K. (2011). Factual argumentation - a core model for assertions making. Journal on Computing and Cultural Heritage, 3(3), 1-34.

Dung, P. M. (1995). On the acceptability of arguments and its fundamental role in nonmonotonic reasoning, logic programming and n-person games. Artificial Intelligence, 77(2), $321-357$

Eco, U. (1990). Travels in Hyperreality (Reprint edition.). San Diego: Mariner Books.

Gardin, J.-C. (2002). Archaeological discourse, conceptual modelling and digitalisation: an interim report of the logicist program. Presented at the Computer Applications and Quantitative Methods in Archaeology 2002.

Gazenbeek, M., Bellavia, V., Braguier, S., Pillard-Jude, C., \& Wiethold, J. (2013). La cuisine d'une maison de maître du Haut-Empire à Grand (Vosges). Gallia Archéologie De La France Antique, 70(1), 97-112.

Hermon, S., \& Kalisperis, L. (2011). Between the Real and the Virtual: 3D visualization in the Cultural Heritage domain - expectations and prospects. Virtual Archaeology Review, 2(4), 59-63.

Hilbert, M., \& López, P. (2011). The world's technological capacity to store, communicate, and compute information. Science, 332(6025), 60-65.

Khanna, S., Tan, W. C., \& Buneman, P. (2001). Why and Where: A Characterization of Data Provenance. Presented at the International Conference on Database Theory 2001.

Kunz, W., \& Rittel, H. W. J. (1970). Issues as elements of information systems.

Le Bœuf, P., Doerr, M., Ore, C. E., \& Stead, S. (2015). CIDOC Conceptual Reference Model

Lopez-Menchero, V. M., \& Grande, A. (2011). The Principles of the Seville Charter (pp. 1-5). Presented at the CIPA symposium 2011.

Marins, A., Casanova, M. A., Furtado, A., \& Breitman, K. (2007). Modeling provenance for semantic desktop applications. Presented at the Online Proceedings for Scientific Workshops 2007.

Missier, P., Belhajjame, K., \& Cheney, J. (2013). The W3C PROV family of specifications for modelling provenance metadata. (pp. 773-776). Presented at the Extending Database Technology 2013.

Oldman, D., Doerr, M., de Jong, G., Norton, B., \& Wikman, T. (2014). Realizing Lessons of the Last 20 Years: A Manifesto for Data Provisioning and Aggregation Services for the Digital Humanities (A Position Paper). D-Lib Magazine, 20(7/8)

Paveprime Ltd. (2014). CRMinf: the Argumentation Model An Extension of CIDOC-CRM to support argumentation.

Pletinckx, D. (2008). An EPOCH Common Infrastructure Tool for Interpretation Management. In Paradata and Transparency in Virtual Heritage.

Reed, C., Walton, D., \& Macagno, F. (2007). Argument diagramming in logic, law and artificial intelligence. The Knowledge Engineering Review, 22(01), 87-109.

Ronzino, P. (2015). CIDOC-CRMBa a CRM Extension for Buildings Archaeology Information Modeling. (Doctoral Dissertation). The Cyprus Institute.

Schuller, M. (2002). Building Archaeology. ICOMOS.

Simmhan, Y., Plale, B., \& Gannon, D. (2005). A survey of data provenance in e-science. ACM Sigmod Record, 34(3), 31-36.

Sweeney, S. (2008). The Ambiguous Origins of the Archival Principle of "Provenance." Libraries \& the Cultural Record, 43(2), 193-213.

Tzompanaki, K., Doerr, M., \& Theodoridou, M. (2013). Reasoning based on property propagation on CIDOC-CRM and CRMdig based repositories. Presented at Practical Experiences with CIDOC CRM and its Extensions (CRMEX 2013) Workshop, 17th International Conference on Theory and Practice of Digital Libraries (TPDL 2013). 